\title{
Ouabain treatment increases nitric oxide bioavailability and decreases superoxide anion production in cerebral vessels Raquel Hernanz ${ }^{\mathrm{a}}$, Ana M. Briones ${ }^{\mathrm{b}}$, Ángela Martín ${ }^{\mathrm{a}}$, Amada E. Beltrán $^{\mathrm{b}}$, Teresa Tejerina $^{c}$, Mercedes Salaices ${ }^{b}$ and María J. Alonso ${ }^{a, b}$
}

Objective Chronic administration of ouabain induces hypertension and increases the contribution of nitric oxide to vasoconstrictor responses in peripheral arteries. The aim of this study was to analyse whether ouabain treatment alters the nitric oxide bioavailability in cerebral arteries.

Methods Basilar arteries from control and ouabain-treated rats $(\sim 8.0 \mu \mathrm{g} /$ day, 5 weeks) were used. Vascular reactivity was analysed by isometric tension recording, protein expression by western blot, nitric oxide levels by diaminofluorescein-induced fluorescence, superoxide anion $\left(\mathrm{O}_{2}^{-\bullet}\right)$ production by ethidium fluorescence and lucigenin chemiluminescence and plasma total antioxidant status by a commercial kit.

Results The relaxations induced by bradykinin $(1 \mathrm{nmol} / \mathrm{l}-10 \mu \mathrm{mol} / \mathrm{l})$ and L-arginine $(0.01-300 \mu \mathrm{mol} / \mathrm{l})$ and the contractile responses induced by both $\mathrm{N}$-nitro-L-arginine methyl ester $(0.1-100 \mu \mathrm{mol} / \mathrm{l})$ and oxyhaemoglobin $(0.01-10 \mu \mathrm{mol} / \mathrm{l})$ were greater in arteries from ouabain-treated than control rats. However, the relaxation to diethylamine NONOate-nitric oxide $(0.1 \mathrm{nmol} / \mathrm{l}-10 \mu \mathrm{mol} / \mathrm{l})$ and the contractions to $\mathrm{KCl}$ $(7.5-120 \mathrm{mmol} / \mathrm{l})$ and 5 -hydroxytryptamine $(0.01-10 \mu \mathrm{mol} / \mathrm{l})$ were similar in arteries from both groups. Ouabain treatment increased basal nitric oxide levels but did not modify endothelial and neuronal nitric oxide synthase protein expression. $\mathrm{O}_{2}{ }^{-\bullet}$ production was lower in cerebral arteries from ouabain-treated rats; however, plasma total antioxidant status and vascular protein expression of $\mathrm{Cu} / \mathrm{Zn}$-superoxide dismutase, Mn-superoxide dismutase and extracellular superoxide dismutase were similar in both groups.

\section{Introduction}

The $\mathrm{Na}^{+}$pump is the major cellular transport system that controls $\mathrm{Na}^{+}$homeostasis and membrane potential, both key factors in the regulation of vascular tone and blood pressure, and thus, alterations in the $\mathrm{Na}^{+}$pump could be an underlying factor in hypertension [1]. Mammalian circulation contains a cardiac glycoside that is putatively of adrenocortical origin and that is structurally similar to the prototypical inhibitor of the sodium pump, ouabain [2]. Circulating levels of this compound as well as ouabain-like immunoreactivity are increased in many forms of hypertension, including essential hypertension $[3,4]$, and there is potential for the development of $\mathrm{Na}^{+}$ pump modulators as antihypertensive drugs [5]. More-
Conclusion Chronic ouabain treatment increased nitric oxide basal levels in basilar arteries probably due to the decreased $\mathrm{O}_{2}^{-}$levels. This might be an adaptive mechanism of the cerebral vasculature to the increase in blood pressure. J Hypertens 26:1944-1954 @ 2008 Wolters Kluwer Health | Lippincott Williams \& Wilkins.

Journal of Hypertension 2008, 26:1944-1954

Keywords: cerebral arteries, hypertension, nitric oxide, ouabain, superoxide anion

Abbreviations: ANOVA, Analysis of variance; cGMP, Cyclic guanosine monophosphate; DAF-2, 4,5-diaminofluorescein diacetate; DEA-nitric oxide, Diethylamine NONOate; DHE, Dihydroethidium; ECF, Enhanced chemifluorescence; $E_{\max }$, Maximum response; eNOS, Endothelial nitric oxide synthase; HEPES, (4-(2-hydroxyethyl)-1-piperazineethanesulfonic acid); iNOS, Inducible nitric oxide synthase; $K_{3} E D T A$, Tripotassium ethylenediamine tetraacetate; KHS, Krebs-Henseleit solution; L-Arg, L-arginine; L-NAME, $N$-nitro-L-arginine methyl ester; $\mathrm{Na}_{2} \mathrm{EDTA}$, Disodium salt of ethylenediaminetetraacetic acid; NADH, Nicotinamide adenine dinucleotide; NADPH, Nicotinamide adenine dinucleotide phosphate; NCX, $\mathrm{Na}^{+}-\mathrm{Ca}^{2+}$-exchanger; nNOS, Neuronal nitric oxide synthase; $\mathrm{O}_{2}{ }^{-}$, Superoxide anion; OxyHb, Oxyhaemoglobin; PI3K, Phosphoinositide 3-kinases; ROS, Reactive oxygen species; SBP, Systolic blood pressure; SDS-PAGE, Sodium dodecyl sulphate-polyacrylamide gel electrophoresis; SOD, Superoxide dismutase; TAS, Total antioxidant status

aDepartamento de Ciencias de la Salud III, Facultad de Ciencias de la Salud Universidad Rey Juan Carlos, ' ${ }^{\mathrm{D}}$ Departamento de Farmacología, Facultad de Medicina, Universidad Autónoma de Madrid and ${ }^{\mathrm{C}}$ Departamento de

Farmacología, Facultad de Medicina, Universidad Complutense de Madrid, Spain

Correspondence to Dr María J. Alonso, PhD, Departamento de Ciencias de la Salud III, Universidad Rey Juan Carlos, Avda. de Atenas, s/n, 28922-Alcorcón, Madrid, Spain

Tel: +34 91 4888875; fax: +3491 4888831; e-mail: mariajesus.alonso@urjc.es

Received 31 October 2007 Revised 4 April 2008 Accepted 14 May 2008

See editorial commentary on page 1901

over, chronic administration of exogenous ouabain is known to induce hypertension in rats [6-14] and mice [15]. Among others, increased sympathetic activity subsequent to the activation of brain renin-angiotensin and endothelin systems and resistance vessel remodelling have all been suggested as playing a role in the development and maintenance of ouabain-induced hypertension $[9,12,16]$. In addition, ouabain enhances basal nitric oxide release by the porcine carotid artery endothelium [17] and human umbilical cord endothelial cells [18]; ouabain-induced hypertension has been described to be accompanied by increased aortic endothelial nitric oxide synthase (eNOS) expression [11] and endothelial nitric oxide modulation of vasoconstrictor 